\title{
Fulvic acid ameliorates drought stress- induced damage in tea plants by regulating the ascorbate metabolism and flavonoids biosynthesis
}

\author{
Jianhao Sun ${ }^{1 \dagger}$, Chen Qiu ${ }^{1 \dagger}$, Yiqian Ding ${ }^{1}$, Yu Wang ${ }^{1}$, Litao Sun ${ }^{1,2}$, Kai Fan¹, Zhongshuai Gai ${ }^{1}$, Guoqiang Dong $^{3}$,
} Jiguo Wang ${ }^{4}$, Xinghui Li ${ }^{5}$, Lubin Song ${ }^{6}$ and Zhaotang Ding ${ }^{1 *}$

\begin{abstract}
Background: Fulvic acid (FA) is a kind of plant growth regulator, which can promote plant growth, play an important role in fighting against drought, improve plant stress resistance, increase production and improve quality. However, the function of FA in tea plants during drought stress remain largely unknown.

Results: Here, we examined the effects of $0.1 \mathrm{~g} / \mathrm{L}$ FA on genes and metabolites in tea plants at different periods of drought stress using transcriptomics and metabolomics profiles. Totally, 30,702 genes and 892 metabolites were identified. Compared with controlled groups, 604 and 3331 differentially expressed metabolite genes (DEGs) were found in FA-treated tea plants at 4 days and 8 days under drought stress, respectively; 54 and 125 differentially expressed metabolites (DEMs) were also found at two time points, respectively. Bioinformatics analysis showed that DEGs and DEMs participated in diverse biological processes such as ascorbate metabolism (GME, AO, ALDH and Lascorbate), glutathione metabolism (GST, G6PDH, glutathione reduced form and CYS-GYL), and flavonoids biosynthesis (C4H, CHS, F3'5'H, F3H, kaempferol, quercetin and myricetin). Moreover, the results of co-expression analysis showed that the interactions of identified DEGs and DEMs diversely involved in ascorbate metabolism, glutathione metabolism, and flavonoids biosynthesis, indicating that FA may be involved in the regulation of these processes during drought stress.

Conclusion: The results indicated that FA enhanced the drought tolerance of tea plants by (i) enhancement of the ascorbate metabolism, (ii) improvement of the glutathione metabolism, as well as (iii) promotion of the flavonoids biosynthesis that significantly improved the antioxidant defense of tea plants during drought stress. This study not only confirmed the main strategies of FA to protect tea plants from drought stress, but also deepened the understanding of the complex molecular mechanism of FA to deal with tea plants to better avoid drought damage.
\end{abstract}

Keywords: Camellia sinensis, Fulvic acid, Drought stress, Ascorbate and glutathione metabolism, Flavonoids biosynthesis

\footnotetext{
* Correspondence: dzttea@163.com

${ }^{\dagger}$ Jianhao Sun and Chen Qiu contributed equally to this work.

${ }^{1}$ Tea Research Institute, Qingdao Agricultural University, Qingdao 266109,

Shandong, China

Full list of author information is available at the end of the article
}

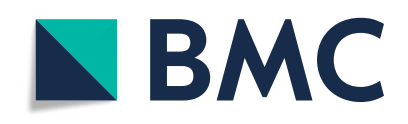

(- The Author(s). 2020 Open Access This article is licensed under a Creative Commons Attribution 4.0 International License, which permits use, sharing, adaptation, distribution and reproduction in any medium or format, as long as you give appropriate credit to the original author(s) and the source, provide a link to the Creative Commons licence, and indicate if changes were made. The images or other third party material in this article are included in the article's Creative Commons licence, unless indicated otherwise in a credit line to the material. If material is not included in the article's Creative Commons licence and your intended use is not permitted by statutory regulation or exceeds the permitted use, you will need to obtain permission directly from the copyright holder. To view a copy of this licence, visit http://creativecommons.org/licenses/by/4.0/ The Creative Commons Public Domain Dedication waiver (http://creativecommons.org/publicdomain/zero/1.0/) applies to the data made available in this article, unless otherwise stated in a credit line to the data. 


\section{Background}

Tea plant (Camellia sinensis (L.) O. Kuntze) is an evergreen crop that likes a humid climate. In field conditions, tea plants are often exposed to drought stress, which has profound effects on tea yield and quality [1]. With the shortage of water resources, the drought has become a main disturbance for tea production in fields, resulting in reduction of yield by around $14-40 \%$ in different cultivation areas every year [2, 3]. In addition, catechins, caffeine, theanine and some free amino acids in tea would be significantly reduced under drought stress, resulting in a serious decline in tea quality [4]. Therefore, exploring the response mechanisms of tea plants to drought stress is essential for developing and breeding new drought-resistant varieties.

Recently, the researches on drought-resistance mechanisms of tea plants have made some progress. In terms of plant morphology, physiology and biochemistry, drought stress could induce stomatal closure, promote the accumulation of proline, glycine betaine, glucose and fructose, and improve the activities of superoxide dismutase and peroxidase [5-8]. In terms of molecular response mechanisms, many drought-responsive genes have been found in tea plants, including heat-shock proteins, glutathione Stransferase genes, sucrose synthases, cytoskeleton proteins and transcription factors [9-12]. However, due to the late start of the study on the mechanism of tea drought resistance, there were few studies on the effects of humic acid application on tea drought resistance under drought stress.

Fulvic acid (FA), an active humic substance, has a relatively low molecular weight and contains a high amount of oxygen-rich and carbon-poor functional groups [13, 14]. Many studies have shown that FA has many plant physiological activities. FA could significantly alleviate the toxic symptoms of Cd on lettuce seedlings [15].. And FA could protect soybean and barley against salt stress [16, 17]. Moreover, FA played important roles in the improvement of drought resistance in plants. Anjum [18] reported that exogenous FA conferred drought tolerance to maize under drought stress by promoting proline contents. And the study of Ramin Lotfi [19] reported that exogenous FA substantially ameliorated the adversities of drought by enhanced levels of superoxide dismutase $(S O D)$, peroxidase $(P O D)$ and catalase $(C A T)$ in rapeseed plants. Regrettably, although these data were valuable for exploring the effects of FA under drought stress, no information had yet been provided on regulatory mechanisms in these studies. It is noteworthy whether the FA could modulate the drought tolerance of tea plants during drought stress.

In the present study, we hypothesis that FA could play positive roles in drought tolerance of tea plants by better enhancing the expression of specific genes and metabolites during drought stress. For this, we examined the effects of $0.1 \mathrm{~g} / \mathrm{L}$ FA on genes and metabolites in tea plants at different periods of drought stress using transcriptomics and metabolomics profiles. These data were valuable for understanding of when and how much antioxidant metabolism should be altered to improve the performance of FA-treated tea plants under drought stress, and would serve as new guiding strategies for improving drought management systems of tea garden.

\section{Results \\ Phenotypic and physiological responses of FA-treated tea plants under drought stress}

In order to verify the effect of FA on tea plants during drought stress, we analyzed the phenotypic and physiological traits of tea shoots under drought stress. The tea plants exhibited the first visible symptom of drought damage, the wilting of shoots, after 4 days in controlled groups (without FA-treated tea plants), while FA-treated tea plants remarkably delayed the wilting of tea shoots. As well as after 8 days of drought, although FA-treated tea plants showed similar trend, the degree of wilting was lower than that of the control (Fig. 1a). Furthermore, the $L W C$ (leaf water content), CC (chlorophyll content), ELC (relative electrolyte conductivity) and ROS (reactive oxygen species) in FA-treated tea plants changed significantly at 4 days of drought stress compared with controlled groups (Fig. 1b). Among them, the $L W C$ and $C C$ in FA-treated tea plants showed downward trends during drought stress, but the extents of the decrease were lower than that of the controlled groups. Furthermore, the ELC and ROS in FA-treated tea plants showed upward trends during drought stress, but the extents of the increase were lower than that of the controlled groups. In addition, after 8 days of drought stress, the differences of $L W C, C C, E L C$ and $R O S$ were largest between FA-treated groups and controlled groups. The results showed that the tea shoots at 4 and 8 days directly reflected the turning point of degree of drought stress. At this time, according to the data of the WHC (water holding capacity of soil), the tea plants were in a state of mild drought $(65-75 \%$ WHC) and severe drought (15-25\% WHC), respectively (Additional file 1). So, we selected the tea shoots of FA-treated groups and controlled groups at 4 days (mild drought) and 8 days (serious drought) as materials to perform transcriptomics and metabolomics analysis.

\section{The transcriptome analysis of FA-treated tea plants under drought stress}

To explore the molecular events of $0.1 \mathrm{~g} / \mathrm{L}$ FA-treated tea plants under drought stress, the transcriptome analysis was performed on tea shoots treated with FA (FAD) and without FA (WD) under drought stress. The samples of 4FAD, 4WD, 8FAD and 8WD were collected and sequenced (Additional file 2). The " 4 " and " 8 " 


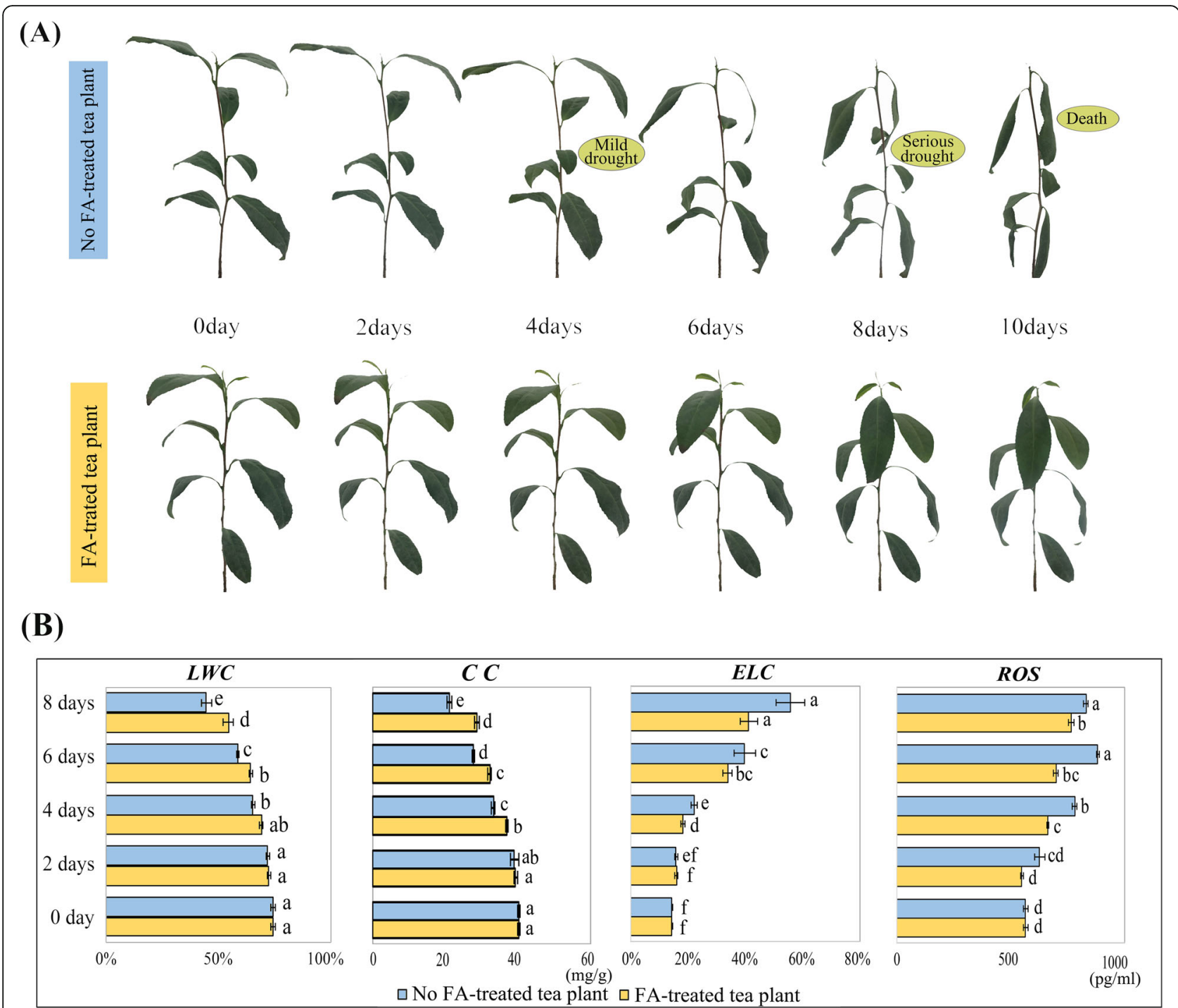

Fig. 1 Phenotypic and physiological traits of FA-treated tea plants under drought stress. a. Phenotypes of FA-treated tea plants and the controlled groups. b. The value of LWC (leaf water content), CC (chlorophyll content), ELC (relative electrolyte conductivity) and ROS (reactive oxygen species)

represented the days of drought stress. After removing the low-quality reads, a total of 537,625,170 clean reads were obtained. The percentages of Q30 and GC were 93.78595.22\% and 44.38-44.83\%, respectively, indicating that the quality of transcriptome sequencing data is high. A total of 30,702 genes were functionally annotated in the databases (Additional file 3). Moreover, 604 (227 up- and 377 downregulated) and 3331 (2149 up- and 1182 down-regulated) DEGs (|fold change $\mid>2$ and corrected $p$-value $<0.05$ ) were identified in the pairwise comparison of 4FADvs4WD and 8FADvs8WD, respectively (Additional file 4). The results indicated that FA could induce the changes of transcripts in tea shoots under drought stress.
The functional analysis of DEGs of FA-treated tea plants under drought stress

To further assess biological functions of DEGs in FAtreated tea plants under drought stress, the Gene Ontology (GO) and Kyoto Encyclopedia of Genes and Genomes (KEGG) enrichment analysis of DEGs were performed (Additional file 5). The results showed that the significantly enriched GO terms of DEGs in 4FADvs4WD were 'secondary metabolic process', 'antioxidant activity', 'fatty acid biosynthetic process' and 'water transport' (Fig. 2a). However, except for 'secondary metabolic process' and 'antioxidant activity', the GO terms of DEGs in 8FADvs8WD were mainly enriched in 


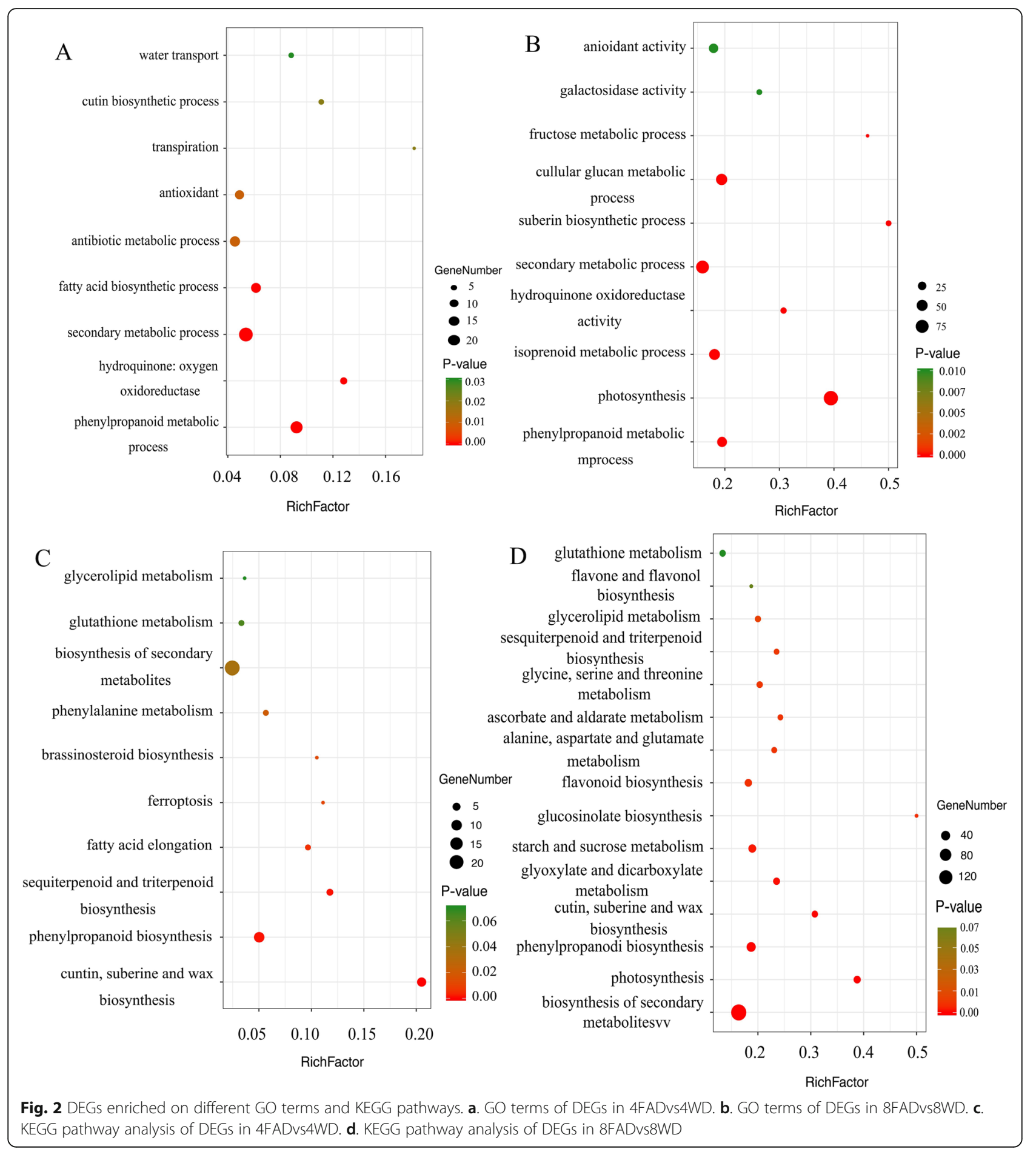

'isoprenoid metabolic process', 'fructose metabolic process' and 'galactosidase activity' (Fig. 2b).

The KEGG pathways of DEGs in 4FADvs4WD and 8FADvs8WD were similar and both included 'phenylpropanoid biosynthesis', 'glutathione metabolism' and 'cutin, suberine and wax biosynthesis' (Fig. 2c and d). In addition, the pathways of DEGs in 4FADvs4WD were also highly enriched in 'phenylalanine metabolism' and 'fatty acid elongation'. While, the high represented pathways of DEGs in 8FADvs8WD were 'flavonoid biosynthesis', 'ascorbate and aldarate metabolism', 'starch and sucrose metabolism' and 'photosynthesis'. The results 
indicated that FA could regulate the complex biological pathways of tea plants under drought stress.

\section{The metabolome analysis of FA-treated tea plants under drought stress}

To further analyze the metabolites of FA-treated tea plants under drought stress, the analysis of widely targeted metabolome was performed using LC-ESI-MS/ MS system. A total of 891 metabolites were obtained in all samples (Fig. 3a, Additional file 6), and clearly separated into four groups according to the PCA analysis (Fig. 3b). Based on a fold-change threshold $>1.5$, the numbers of up-regulated and down-regulated metabolites in 4FADvs4WD and 8FADvs8WD were 21 and 32, 34 and 90, respectively (Additional file 7). These differentially expressed metabolites (DEMs) were summarized into 143 species, which were mainly classified into flavonoid (2), vitamins and derivatives (3), flavonol (6), flavone (7), phenylpropanoids (15), organic acids and derivatives (24) and others (Additional file 8). Interestingly, the DEMs associated with osmotic adjustment, such as proline, glucose and fructose, were not found to accumulate significantly in 4FADvs4WD and 8FADvs8WD. However, whether in 4FADvs4WD or in 8FADvs8WD,

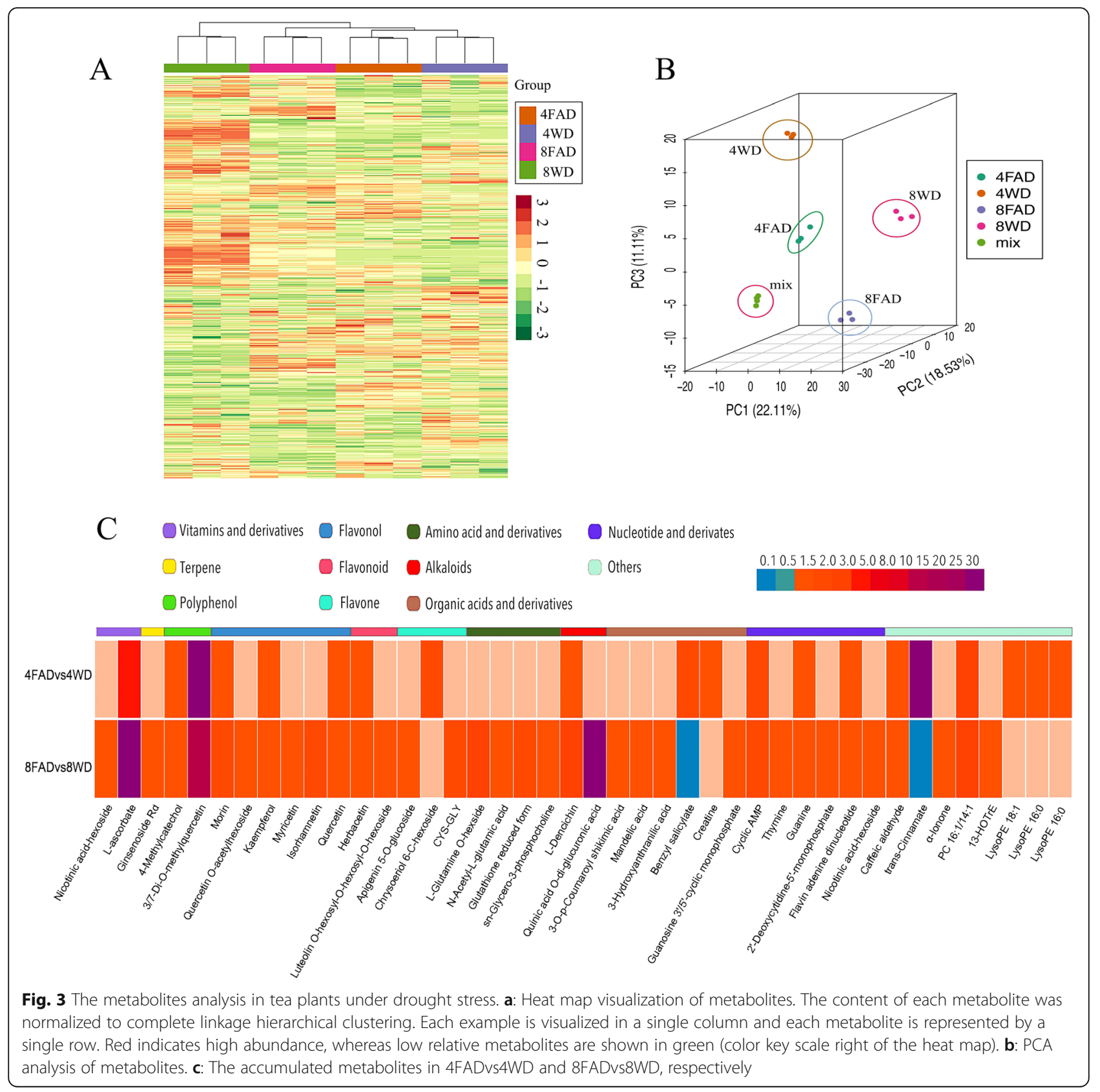


the DEMs related to ascorbate metabolism and flavonoids biosynthesis were all highly accumulated. In addition, the more DEMs related to flavonoids biosynthesis were found in 8FADvs8WD and the DEMs related to glutathione metabolism were also accumulated (Fig. 3c). The results showed that FA could induce the changes of antioxidants in tea plants under drought stress.

\section{The co-expression network analysis of DEGs and DEMs in} FA-treated tea plants under drought stress

In order to examine the relationship of DEGs and DEMs in FA-treated tea plants during drought stress, the co-expression network analysis of DEGs and DEMs was conducted (Pearson correlation coefficient $>0.8$ or $<-0.8, P$-value $<0.05$; Additional file 9). The DEGs and DEMs in 4FADvs4WD, such as caffeoyl-CoA Omethyltransferase, Quercetin and 3,7-Di-O-methylquercetin, were found to be related to flavonoids biosynthesis (Fig. 4a). While, except for flavonoids biosynthesis (e.g. Chalcone synthase 3 , Chalcone synthase 1, flavanone 3-hydroxylase, flavonoid 3',5'-hydroxylase, Quercetin, Kaempferol, Myricetin, Phloretin and Myricetin) (Fig. 4b), the co-expression network of DEGs and DEMs in 8FADvs8WD were mainly enriched in ascorbate metabolism (e.g. L-ascorbate oxidase, aldehyde dehydrogenase family 3, GDP-mannose-3',5'-epimerase perakine reductas, L-ascorbate) (Fig. 4c) and glutathione metabolism (e.g. glucose-6-phosphate 1-dehydrogenase 1, glutathione S-transferase, CYS-GLY and Glutathione reduced form) (Fig. 4d). The results showed that the FA could modulate the co-expression of DEGs and DEMs related to ascorbate metabolism, glutathione metabolism and flavonoids biosynthesis during drought stress.

The integrated analysis of genes and metabolites related to ascorbate metabolism in FA-treated tea plants under drought stress

To check into the impacts of FA on genes and metabolites related to ascorbate biosynthesis in tea plants under drought stress, the interaction of DEGs and DEMs related to ascorbate metabolism was analyzed (Fig. 5a, Additional file 10). The DEGs were not found to be related to ascorbate metabolism in 4FADvs4WD, while 8 DEGs were found in 8FADvs8WD. Of which, the DEGs, such as GME (GDP-mannose-3', $5^{\prime}$-epimerase, TEA005483), $A L D H$ (aldehyde dehydrogenase family 3, TEA032018) and $P R$ (perakine reductase, TEA011544) were up-regulated, and $A O$ (L-ascorbate oxidase, TEA018652 and TEA018659) were down-regulated. Moreover, L-ascorbate, the downstream metabolite related to ascorbate metabolism, was found to be significantly accumulated both in 4FADvs4WD and 8FADvs8WD. The results showed that the DEGs and DEMs related to ascorbate metabolism in FA-treated tea plants were taken conjointly to respond to mild drought stress and serious drought stress.

The integrated analysis of genes and metabolites related to glutathione metabolism in FA-treated tea plants under drought stress

To dig out the impacts of FA on expressions of genes and metabolites related to glutathione metabolism in tea plants under drought stress, the interaction of DEGs and DEMs related to glutathione metabolism was analyzed (Fig. 5b, Additional file 11). The 3 and 12 DEGs were found to be related to glutathione metabolism in 4FADvs4WD and 8FADvs8WD, respectively. Of them, 2 GST genes (glutathione S-transferase, TEA011287 and TEA003225) were up-regulated in 4FADvs4WD, and GST (TEA015341) was down-regulated. Furthermore, in 8FADvs8WD, 6 GST genes (TEA000526, TEA000598, TEA010114, TEA010988, TEA022693, TEA026775 and TEA028520) and G6PDH (glucose-6-phosphate 1dehydrogenase 1, TEA024651) were up-regulated. Moreover, the glutathione reduced form and CYS-GLY, the downstream metabolite related to glutathione metabolism, were found to be accumulated only in 8FADvs8WD. The results showed that the DEGs and DEMs related to glutathione metabolism in FA-treated tea plants were taken conjointly to respond to mild drought stress and serious drought stress.

The integrated analysis of genes and metabolites related to flavonoids biosynthesis in FA-treated tea plants under drought stress

To probe into the impacts of FA on genes and metabolites related to secondary metabolism in tea plants under drought stress, the interaction of DEGs and DEMs related to flavonoids biosynthesis was analyzed (Fig. 6, Additional file 12). The 2 DEGs related to flavonoids biosynthesis, C4H (cinnamate-4-hydroxylase, TEA016772) and CCM (caffeoyl-CoA O-methyltransferase, TEA030958), were found to be upregulated in 4FADvs4WD. Of which, the, were up-regulated. Furthermore, 20 DEGs were found in 8FADvs8WD, such as F3'5'H (flavonoid 3',5'-hydroxylase, TEA013315), LAR (leucoanthocyanidin reductase, TEA027582), F3H (flavanone 3-hydroxylase, TEA023790), CHI (chalcone isomerase, TEA034003), CHS1(chalcone synthase 1, TEA023340) and CHS3 (chalcone synthase 3, TEA018665) were up-regulated. Moreover, the kaempferol and quercetin, the downstream metabolite related to flavonoids biosynthesis, were found to be accumulated both in 4FADvs4WD and 8FADvs8WD. And the myricetin was accumulated only in 8FADvs8WD. The results showed that the DEGs and DEMs related to flavonoids biosynthesis in FA-treated tea plants were taken conjointly to respond to mild drought stress and serious drought stress. 


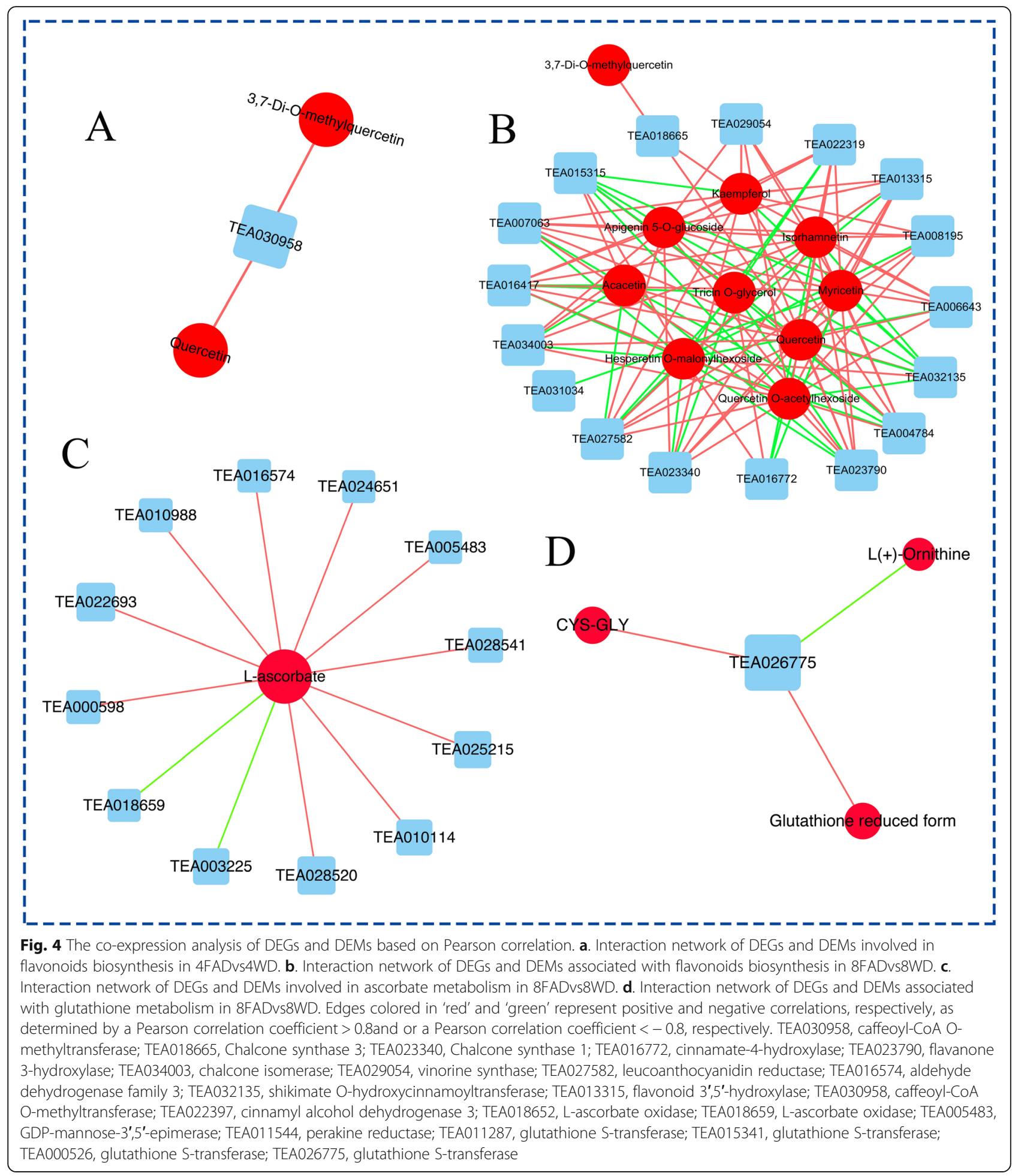

The qRT-PCR validation of DEGs in FA-treated tea plants under drought stress

To test the accuracy of our RNA-Seq data, quantitative RT-PCR (qRT-PCR) was performed on 10 DEGs selected from the ascorbate metabolism, glutathione metabolism and flavonoids biosynthesis, such as $A O, F 3 H, F 3{ }^{\prime}{ }^{\prime} H$ and $L A R$. The RNA-seq (FPKM) and qRT-PCR analyses were similar (Additional file 13), showing the same general expression trends (more than 90\% correlation). These results verified the reproducibility and credibility of RNA-seq data. 


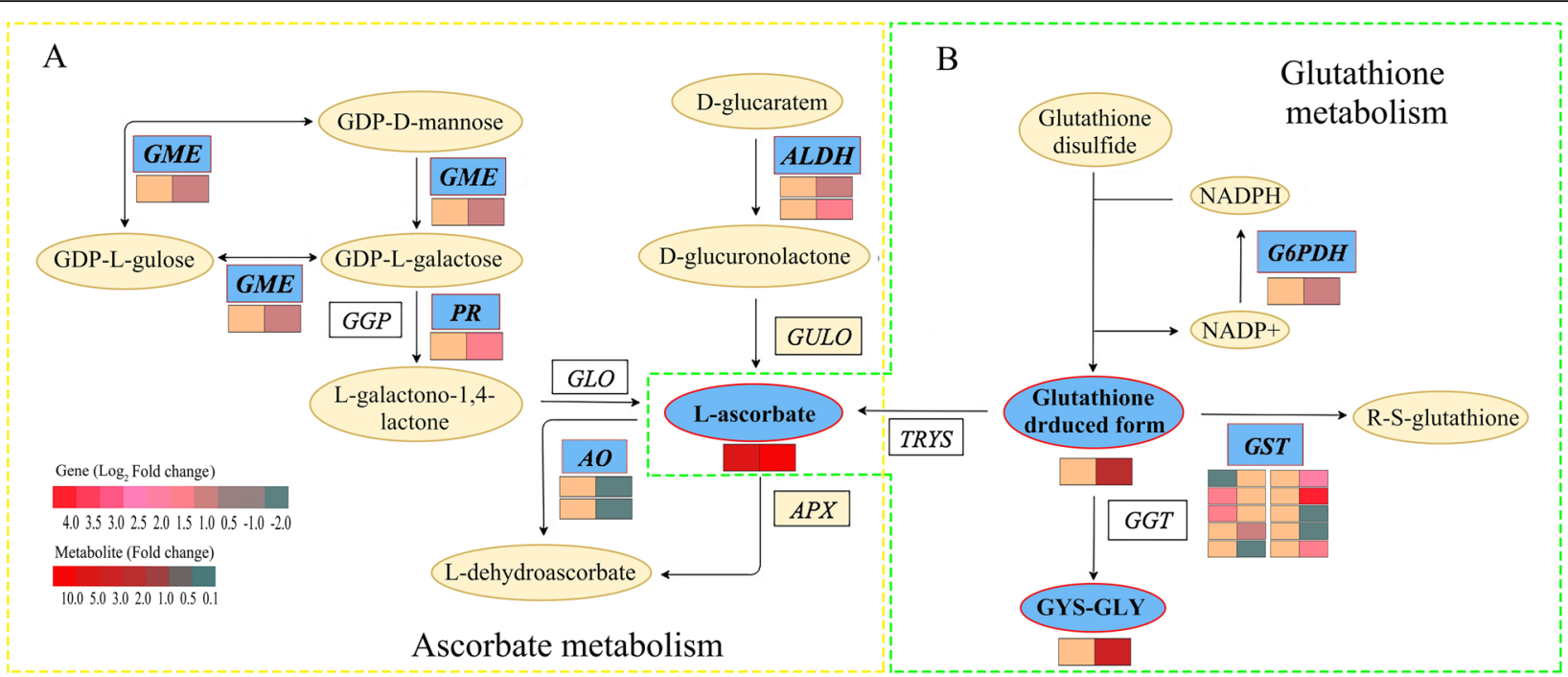

Fig. 5 The DEGs and DEMs involved in ascorbate and glutathione metabolism in response to drought stress. a. The DEGs and DEMs involved in ascorbate metabolism. $\mathbf{b}$. The DEGs and DEMs involved in glutathione metabolism. The yellow dotted box on the left represented the ascorbate metabolism process, and the green dotted box on the right represented the glutathione metabolism. The blue pattern represented the metabolites or genes that changed under drought stress. The rectangle was divided into two equal parts (the left of rectangle represented DEGs or DEMs in 4FADvs4WD; the right of rectangle represented DEGs or DEMs in 8FADvs8WD). The color in the rectangle represents the genes or metabolites were regulated under drought stress (red indicated up-regulation; yellow indicates; green indicated down-regulation). GME: GDPmannose-3',5'-epimerase; AO: L-ascorbate oxidase; ALDH: aldehyde dehydrogenase; GST: glutathione S-transferase; PR: perakine reductase; G6PDH: glucose-6-phosphate 1-dehydrogenase

\section{Discussion}

In the study, to explore the roles of FA in tea plants under drought condition, we studied the effects of $0.1 \mathrm{~g} /$ L FA on ascorbate metabolism, glutathione metabolism and flavonoids biosynthesis by the integrated analysis of transcriptome and metabolome. The results showed that FA could ameliorate drought stress-induced damage in tea plants.

\section{FA enhanced the ascorbate metabolism in tea plants under drought stress}

The drought stress often causes rapid and excessive accumulation of ROS in plant cells, which resulted in degradation of chlorophyll, lipid peroxidation, enzyme inactivation and DNA damage, and eventually led to metabolic disorder and apoptosis [20, 21]. To protect the plants from oxidative stress and maintain normal cellular functions, plants in drought condition would scavenge excess $R O S$ through $R O S$ scavenging enzymes $(C A T, P O D$ and $S O D)$ and non-enzymatic antioxidants (including ascorbate and glutathione) [22, 23]. Interestingly, in the present study, the $S O D, C A T$ and $P O D$ did not show significant statistical difference $(P>0.05)$ at the level of transcription. The results hinted that FA might improve the ROS scavenging of tea plants mainly by enhancing the biosynthesis of non-enzymatic antioxidants.
Ascorbic acid functions as an antioxidant and enzymatic cofactor and plays effective roles in maintenance of $R O S$ homeostasis [24]. However, up to now, the ascorbate in FA-treated plants under drought stress has not been reported. In this study, the contents of ascorbate were accumulated both in 4FADvs4WD and 8FADvs8WD, and the statistically differences under serious drought stress were severe (fold changes $>30$ ). In addition, the GME and $A O$ were up-regulated and down-regulated in 8 FADvs $8 \mathrm{WD}$, respectively. The GME, generally considered to be a central enzyme of ascorbate biosynthesis pathway, catalyzed the conversion of GDP-D-mannose to GDP-L-galactose in the D-mannose/L-galactose pathway [25]. The $A O$ could catalyze the oxidation of ascorbate to the unstable radical monodehydroascorbate which rapidly disproportionated to yield dehydroascorbate [26]. Previous studies showed that the GME could effectively enhance tolerance of transgenic Arabidopsis to drought by increasing ascorbate accumulation [25]. The $A O$ was involved in the negative regulation of the stress response, and $A O$ caused oxidation of ascorbate, thereby preventing the detoxification of $R O S$ [27]. Therefore, we deduced that FA might enhance the ROS homeostasis of tea plants during drought stress by improving the biosynthesis of ascorbate, while further enhance the ROS homeostasis under serious drought stress by improving the expression of $G M E$ and restraining the expression of $A O$. 


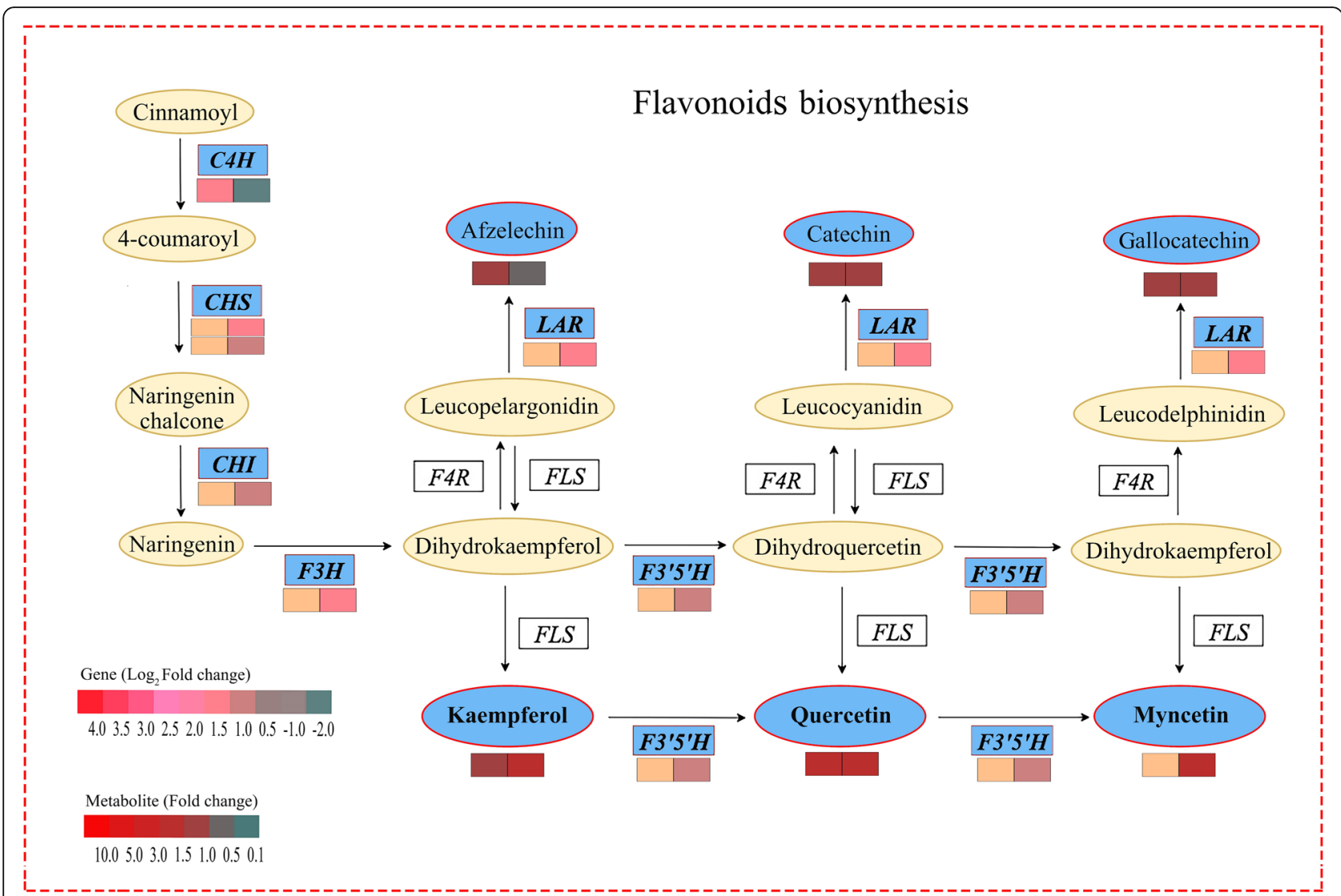

Fig. 6 The DEGs and DEMs involved in flavonoids biosynthesis in response to drought stress. The blue pattern represented the metabolites or genes that changed under drought stress. The rectangle was divided into two equal parts (the left of rectangle represented DEGs or DEMs in 4FADvs4WD; the right of rectangle represented DEGs or DEMs in 8FADvs8WD). The color in the rectangle represents the genes or metabolites were regulated under drought stress (red indicated up-regulation; green indicated down-regulation). C4H: cinnamate-4-hydroxylase; CHS; chalcone synthase; CHI: chalcone isomerase; F3H: flavanone 3-hydroxylase; F3'5'H: flavonoid 3',5'-hydroxylase; LAR: leucoanthocyanidin reductase

\section{FA enhanced the glutathione metabolism in tea plants under drought stress}

Glutathione is one of the major endogenous antioxidants in plants, which functions as a substrate in antioxidative defense mechanisms by conjugating to toxic electrophilic compounds, scavenging free radicals, and reducing peroxides $[28,29]$. Previous studies showed the G6PDH was involved in glutathione content maintenance and $R O S$ scavenging under drought stress by utilizing NADPH [30]. The expression of G6PDH in tomato could control the pace of ROS increment and improve the drought tolerance [31]. In this study, the G6PDH and glutathione reduced form were accumulated only in 8 FADvs8WD, indicating that the FA might improve the ROS scavenging in tea plants under serious drought stress by improving the expression of $G 6 P D H$ and the biosynthesis of glutathione reduced form. In addition, previous studies showed that GST was enzyme that utilize glutathione to play an important role in plant defense metabolism by mechanisms that aid in the reduction of secondary noxious products resulting from exposure to stressinduced ROS $[28,32]$. And the over-expressing GST improved drought tolerance in transgenic Arabidopsis [33]. In the study, we also found that GST genes were upregulated both in 4FADvs4WD and 8FADvs8WD. Therefore, we speculated that FA might reduce the secondary injury products of $R O S$ under drought stress through the expression of GST.

\section{FA improved the flavonoids biosynthesis in tea plants under drought stress}

Flavonoids are a group of polyphenol compounds with known antioxidant activities, which are composed of flavones, flavonols, flavanones, anthocyanins and isoflavones [34]. It has been suggested that the accumulation of flavonoids could be a key step in development of plant tolerance to different environmental stresses [35]. The $\mathrm{C} 4 \mathrm{H}$, a member of the cytochrome P450 monooxygenase superfamily, controlled the synthesis of p-coumaric acid from transcinnamic acid [36]. The CHS catalyzed the condensation of 
three molecules of malonyl-CoA and one molecule of 4coumaroyl-CoA to naringenin chalcone, which was the substrate for $\mathrm{CHI}$ and was converted to naringenin [37]. Earlier researches revealed that the $\mathrm{C} 4 \mathrm{H}$ involved in the drought defense of cucumber [38]. And the CHS from Abelmoschus esculentus regulated flavonoid accumulation and abiotic stress tolerance in transgenic Arabidopsis [39]. In this study, we found that the $\mathrm{C} 4 \mathrm{H}$ and $2 \mathrm{CHS}$ genes (CHS1 and CHS3) were up-regulated in 4FADvs4WD and 8FADvs8WD, respectively. Therefore, we speculated that FA could modulate the drought tolerance of tea plants during drought stress by improving the expression of $\mathrm{C} 4 \mathrm{H}$ and CHS in mild drought and serious drought, respectively.

The main physiological functions of quercetin, kaempferol and myricetin in plants were scavenging reactive oxygen species and acting to detoxification of free radicals, which increasing tolerance to adapt to environmental change [40-42]. Previous study showed that the accumulation of quercetin and kaempferol under water deficit could improve the drought tolerance of white clover [43]. However, the information of flavonoids in FA-treated plants under drought stress was rare. In this study, the flavonoids, including kaempferol, quercetin and myricetin, were accumulated during drought stress, and the corresponding genes, including $F 3^{\prime} H$ and $F 3^{\prime} 5^{\prime} H$, were up-regulated in 8FADvs8WD. Previous studies showed that the $F 3^{\prime} H$ and $F 3^{\prime} 5^{\prime} H$ played a key role in affecting the composition of dihydroxylated and trihydroxylated flavonoids [44]. And the lack of expression of the $F 3^{\prime} 5^{\prime} H$ in the grapes restricted the presence of quercetin, kaempferol, myricetin and syringetin derivatives [45]. Thus, we have reason to deduce that the FA might enhance the ROS scavenging by improving the biosynthesis of kaempferol, quercetin and myricetin during drought stress, while further enhance $R O S$ scavenging by the expression of $F 3^{\prime} H$ and $F 3^{\prime}{ }^{\prime} ' H$ under serious drought stress.

The $L A R$ catalyzes the conversion of leucocyanidin, leucodelphinidin or leucopelargonidin to the corresponding flavan-3-ol units (catechin and gallocatechin) [46]. And the overexpression of $L A R$ in plants often results in the increases of catechin and epicatechin [47]. According to previous report in berry, the soil drought could induce the expression of $L A R$ genes, resulted in the accumulation of catechins [48]. The results from the current paper indicated that the $L A R$ was up-regulated in 8FADvs8WD, and the catechin and gallocatechin were also slightly increased in FA-treated tea plants. Since previous studies have shown that amongst flavonoids, flavan-3-ols (catechin, epicatechin and gallocatechin) have direct free radical scavenging activity to maintain the normal physiological function of cells in vivo [49]. So, these data meant that FA might contribute to the drought acclimation of tea plants under serious drought condition by improving the expression of $L A R$ and the biosynthesis of catechin and gallocatechin.

\section{Conclusion}

In the present study, we investigated the positive roles of FA in tea plants under drought stress. The novel regulatory and functional candidates in ascorbate metabolism, glutathione metabolism and flavonoids biosynthesis in FA-treated tea plants in providing drought tolerance were successfully identified by comparative transcriptional and metabolic analysis. Our study confirmed that the primary strategy for FA to protect tea plants against drought-stress was to enhance the detoxifying $R O S$ and regulate the antioxidant systems.

\section{Methods}

\section{Plant material and experimental design}

The uniformly sized one-year-old seedlings of C.sinensis (L.) O. Kuntze cv. 'QN3' [50], acquired from Qingdao Agricultural University in Shandong Province of China $\left(36^{\circ} 19^{\prime} \mathrm{N}, 120^{\circ} 23^{\prime} \mathrm{E}\right)$, were transplanted into plastic cups filled with sandy loam soil and $\mathrm{pH}$ 4.5. The 'QN3' was identified by Prof Zhaotang Ding, Yu wang and etc. And it has been deposited in the national germplasm tea repository (Hangzhou, China). The seedlings were culture-grown under a $12 \mathrm{~h}$ light $\left(25^{\circ} \mathrm{C}\right) / 12 \mathrm{~h}$ dark $\left(18^{\circ} \mathrm{C}\right)$ photoperiod with $300 \mu \mathrm{M} \cdot \mathrm{m}-2 \cdot \mathrm{s}-1$ photon flux densities and 75\% humidity. After 1 week, the tea plants in plastic cups subjected to drought stress. The preliminary experiments for FA concentration screening (0.01, $0.05,0.1,0.5$ and $1 \mathrm{~g} / \mathrm{L} F A)$ were found that $0.1 \mathrm{~g} / \mathrm{L} F A$ was better concentration for alleviating drought damage during water deficit (See Additional file 14 for a detailed description). The FA was purchased from Bio Dibai (Shanghai, China). In the formalized testing, tea plants in plastic cups were exposed to drought stress after irrigating $15 \mathrm{ml}$ of $0.1 \mathrm{~g} / \mathrm{L} \mathrm{FA}$, and the same water was irrigated as the control. The soil water content in the normal condition was at $65-75 \%$ water holding capacity, the severe drought condition was at $15-25 \%$ water holding capacity [51]. The $L W C, R E L$ and ROS contents of tea new shoots (the sprout and the two leaves below) were assayed during drought stress. Based on the results of trials, the tea shoots were harvested at 4 (mild drought stress) and 8 (severe drought stress) days of drought stress for analyzing the transcriptome and metabolome. Three biological replicates for each sampling time point were collected and immediately stored at $-80^{\circ} \mathrm{C}$.

\section{Measurement of physiological indices of drought stress}

In physiological experiments, more than five tea plants were harvested for each treatment group at $0,2,4,6,8$, and 10 days. The tea shoots were used to test the physiological traits containing $L W C, E L C$ and ROS. The $L W C$ and $E L C$ were determined as described previously [52, 53]. The ROS was tested using ELISA kit (Jiangsu Meimian, China). 


\section{Transcriptome sequencing and data analysis}

The samples of 4FAD, 4WD, 8FAD and 8WD were selected for total RNA extract, which using the Trizol reagent (Invitrogen, USA). Refer to previous study for transcriptome sequencing [54]. Briefly, RNA concentrations were measured by Qubit 2.0 Flurometer (Life Technologies, USA). The cDNA library was prepared for sequencing using the NEBNext Ultra RNA Library Prep Kit. The prepared library was sequenced using novaseq 6000 platform. Reads containing adapters, sequences with more than $10 \%$ unknown nucleotides $(\mathrm{N})$, and a quality rating less than $50 \%$ (Q-value $\leq 20$ ) were first removed from the data set. Index of the reference genome was built using Bowtie v2.2.3 and paired-end clean reads were aligned to the tea plant reference genome (http://tpia.teaplant.org/) using TopHat (v2.0.12). HTSeq v0.6.1 was used to count the reads numbers mapped to each gene. And then FPKM of each gene was calculated based on the length of the gene and reads count mapped to this gene. Differential expression between two conditions/groups was analysed with the DESeq R package (1.10.1). Genes with $|\log 2 \mathrm{FC}|>1$ and $p$ value $<0.05$ were considered as DEGs. To infer the putative functions of DEGs, we conducted GO functions and KEGG pathway analysis by the OmicShare tools (http://www. omicshare.com/tools).

\section{Metabolite profiling analysis}

The extract analysis, metabolite identification and quantification were performed by MetWare (Wuhan, China) following their standard procedures and previous study [55]. Metabolite data analysis was conducted with the Analyst 1.6.3 software. Metabolites with fold change $\geq 1.5$ were considered as DEMs.

\section{Co-expression network analysis of metabolome and transcriptome}

The EXCEL program was used to calculate the Pearson correlation coefficients according to the fold changes of each DEGs and each DEMs. Correlations corresponding to a coefficient with $R^{2}>0.8$ were selected. The Cytoscape (version 2.8.2) was used to visualize the relationship between metabolome and transcriptome.

\section{The qRT-PCR validation for DEGs}

The qRT-PCR analyses were conducted with mRNA from the tea shoots. The method followed the procedures described in a previous report [56]. The triplicates of each reaction were performed, and GAPDH sequence was used as endogenous control. CT values obtained through qRT-PCR were analyzed using $2^{-\Delta C T}$ method to calculate relative fold change values [57]. The primers for the qRT-PCR are described in Additional file 15.

\section{Supplementary information}

Supplementary information accompanies this paper at https://doi.org/10. 1186/s12864-020-06815-4

Additional file 1. The water holding capacity of soil.

Additional file 2. The information of transcriptomes.

Additional file $\mathbf{3}$. The annotation of total reads.

Additional file 4. The information of DEGs in 4FADvs4WD and 8FADvs8WD

Additional file 5. The information of $\mathrm{GO}$ and $\mathrm{KEGG}$ analysis of DEGs.

Additional file 6. The information of metabolomes.

Additional file 7. The information of DEMs in 4FADVs4WD and 8FADvs8WD.

Additional file 8. The summary of DEMs in 4FADvs4WD and 8FADvs8WD.

Additional file 9. The information of co-expression network of DEGs and DEMs.

Additional file 10. The information of DEGs and DEMs involved in ascorbate metabolism.

Additional file 11. The information of DEGs and DEMs involved in glutathione metabolism.

Additional file 12. The information of DEGs and DEMs involved in flavonoids biosynthesis.

Additional file 13. The qRT-PCR validation of 10 genes.

Additional file 14. The detailed description for concentration screening of fulvic acid under drought stress.

Additional file 15. The information of primers used for qRT-PCR.

\section{Abbreviations}

FA: Fulvic acid; SOD: Superoxide dismutase; POD: Peroxidase; CAT: Catalase; LWC: Relative water content; CC: Chlorophyll content; ELC: Relative electrolyte conductivity; ROS: Reactive oxygen species; FAD: FA-treated tea plants were exposed to drought stress; 4FAD: FA-treated tea plants at 4 days of drought stress; 8FAD: FA-treated tea plants at 8 days of drought stress; 4WD: The controlled groups at 4 days of drought stress; 8WD: The controlled groups at 8 days of drought stress; LC-MS/MS: liquid chromatography tandem mass spectrometry; RNA-seq: RNA sequencing; GO: Gene Ontology; KEGG: Kyoto Encyclopedia of Genes and Genomes; DEGs: Differentially expressed genes; DEMs: Differentially expressed metabolites; qRT-PCR: Quantitative RT-PCR; GME: GDP-mannose-3',5'-epimerase; AO: L-ascorbate oxidase; ALDH: Aldehyde dehydrogenase; PR: Perakine reductase; G6PDH: Glucose-6-phosphate 1dehydrogenase; GST: Glutathione S-transferase; $C 4 H$ : Cinnamate-4hydroxylase; CHS: Chalcone synthase; CHI: Chalcone isomerase; F3H: Flavanone 3-hydroxylase; F3'5'H: Flavonoid 3',5'-hydroxylase; LAR: Leucoanthocyanidin reductase

\section{Acknowledgements}

We would like to thank all authors for valuable discussions.

\section{Authors' contributions}

CQ and JS, carried out the experiment, collected and organized data and wrote the manuscript. YD, YW, LS1, KF and ZG participated in designing the experiment and directed the study. $\mathrm{XL}, \mathrm{LS} 2, \mathrm{GD}$ and JW, reviewed the manuscript and organized the data. ZD, corresponding author, raised the hypothesis underlying this work, designed the experiment, and helped organize the manuscript structure. All authors read and approved the final manuscript.

\section{Funding}

This work was supported by the Breeding Project of Shandong Province (2017LZN014), the Significant Application Projects of Agriculture Technology Innovation in Shandong Province (SD2019ZZ010), the Technology System of Modern Agricultural Industry in Shandong Province (SDAIT-19-01), the Special Foundation for Distinguished Taishan Scholar of Shandong Province (ts201712057), the Key Research and Development Project of Shandong 
province (2019LYXZ009). The funds played no role in study design, data analysis, and manuscript preparation.

\section{Availability of data and materials}

The datasets supporting the results of this article are available at the Sequence ReadArchive (SRA) database of National Center for Biotechnology Information (NCBl; https://www.ncbi.nlm.nih.gov/) under project accession number PRJNA596070.

\section{Ethics approval and consent to participate}

Not applicable. The tea plants used in this study were provided by Tea Research Institute of Qingdao Agricultural University. It does not require ethical approval.

\section{Consent for publication}

Not applicable.

\section{Competing interests}

The authors declare that they have no competing interests.

\section{Author details}

${ }^{1}$ Tea Research Institute, Qingdao Agricultural University, Qingdao 266109 , Shandong, China. ${ }^{2}$ Murdoch University, Perth 6150, Australia. ${ }^{3}$ The Quality and Safety Center of Agricultural Products, Qingdao 266000, Shandong, China. ${ }^{4}$ The Service Center of Agricultural Technology, Rizhao 276800, Shandong, China. ${ }^{5}$ Tea Research Institute, Nanjing Agricultural University, Nanjing 210095, Jiangsu, China. Institue of Pomology, Shandong Academy of Agricultural Sciences, Taian 271000, Shandong, China.

\section{Received: 20 December 2019 Accepted: 9 June 2020}

Published online: 18 June 2020

\section{References}

1. Han WY, Li X, Ahammed GJ. Stress physiology of tea in the face of climate change; 2018.

2. Gupta S, Bharalee R, Bhorali P, Das SK, Bhagawati P, Bandyopadhyay T, Gohain B, Agarwal N, Ahmed P, Borchetia S, et al. Molecular analysis of drought tolerance in tea by CDNA-AFLP based transcript profiling. Mol Biotechnol. 2013;53(3):237-48.

3. Das A, Das S, Mondal TK. Identification of differentially expressed gene profiles in young roots of tea [Camellia sinensis (L.) O. Kuntze] subjected to drought stress using suppression subtractive hybridization. Plant Mol Biol Report. 2012;30(5):1088-101.

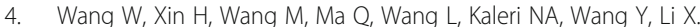
Transcriptomic analysis reveals the molecular mechanisms of droughtstress-induced decreases in Camellia sinensis leaf quality. Front Plant Sci. 2016;7:385.

5. Liu SC, Yao M-Z, Ma C-L, Jin J-Q, Ma J-Q, Li C-F, Chen L. Physiological changes and differential gene expression of tea plant under dehydration and rehydration conditions. Sci Hortic. 2015;184:129-41.

6. Das A. Influence of drought stress on cellular ultrastructure and antioxidant system in tea cultivars with different drought sensitivities. J Environ Biol. 2015;36(36):875-82.

7. Netto LA, Jayaram KM, Puthur JT. Clonal variation of tea [Camellia sinensis ( $L$. ) O. Kuntze] in countering water deficiency. Physiol Mol Biol Plants. 2010; 16(4):359-67.

8. Mukhopadhyay M, Mondal TK. The physico-chemical responses of Camellia to abiotic stresses. J Plant Sci Res. 2014;1:105.

9. Muoki RC, Paul A, Kumar S. A shared response of thaumatin like protein, chitinase, andlate embryogenesis abundant protein 3 to environmental stresses in tea [Camellia sinensis (L.) O. Kuntze]. Functional Integrative Genomics. 2012;12(3):565-71.

10. Wang Y, Fan K, Wang J, Ding Z-t, Wang H, Bi C-h, Zhang Y-w, Sun H-W. Proteomic analysis of Camellia sinensis ( $\mathrm{L}$.) reveals a synergistic network in the response to drought stress and recovery. J Plant Physiol. 2017;219:91-9.

11. Gupta S, Bharalee R, Bhorali P, Bandyopadhyay T, Gohain B, Agarwal N, Ahmed P, Saikia H, Borchetia S, Kalita MC. Identification of drought tolerant progenies in tea by gene expression analysis. Functional Integrative Genomics. 2012;12(3):543-63.

12. Mukhopadhyay M, Mondal TK, Chand PK. Biotechnological advances in tea (Camellia sinensis [L.] O. Kuntze): a review. Plant Cell Rep. 2016;35(2):255-87.
13. Dong L, Yuan Q, Yuan H. Changes of chemical properties of humic acids from crude and fungal transformed lignite. Fuel. 2006;85(17-18):2402-7.

14. Tate RL. Humic substances in soil and crop sciences. Soil Sci. 1991;152(3):237.

15. Wang Y, Yang R, Zheng J, Shen Z, Xu X. Exogenous foliar application of fulvic acid alleviate cadmium toxicity in lettuce (Lactuca sativa L.). Ecotoxicology Environ Safety. 2019;167:10-9.

16. Jarošová M, Bi K, Kováčik J, Babula P, Hedbavny J. Humic acid protects barley against salinity. Acta Physiol Plant. 2006;38(6):161.

17. Dinler BS, Gunduzer E, Tekinay T. Pre-treatment of fulvic acid plays a stimulant role in protection of soybean (Glycine max L.) leaves against heat and salt stress. Acta Biologica Cracoviensia s Botanica. 2016;58(1):29-41.

18. Anjum SA, Wang L, Farooq $M$, Xue L, Ali S. Fulvic acid application improves the maize performance under well-watered and drought conditions. J Agronomy Crop Sci. 2011;197(6):409-17.

19. Lotfi R, Pessarakli M, Gharavi-Kouchebagh P, Khoshvaghti H. Physiological responses of Brassica napus to fulvic acid under water stress: chlorophyll a fluorescence and antioxidant enzyme activity. Crop J. 2015;5:434-9.

20. Bałabusta M, Szafrańska K, Posmyk MM. Exogenous melatonin improves antioxidant defense in cucumber seeds (Cucumis sativus L.) germinated under chilling stress. Front Plant Sci. 2016;7:575.

21. Allakhverdiev SI, Kreslavski VD, Klimov W, Los DA, Carpentier R, Mohanty P. Heat stress: an overview of molecular responses in photosynthesis. Photosynth Res. 2008;98(1-3):541-50.

22. Zhang H, Zhang N, Yang R-C, Wang L, Sun Q, Li D-B, Cao Y-Y, Weeda S, Zhao B, Ren $\mathrm{S}$, et al. Melatonin promotes seed germination under high salinity by regulating antioxidant systems, $A B A$ and GA4 interaction in cucumber (Cucumis sativus L.). J Pineal Res. 2014;57(3):269-79.

23. Sharif R, Xie C, Zhang H, Arnao MB, Ali M, Ali Q, Muhammad I, Shalmani A, Nawaz MA, Chen $P$, et al. Melatonin and its effects on plant systems. Molecules. 2018:23:2352.

24. Conklin PL, Barth C. Ascorbic acid, a familiar small molecule intertwined in the response of plants to ozone, pathogens, and the onset of senescence. Plant Cell Environ. 2004;27:959-70.

25. Ma L, Wang Y, Liu W, Liu Z. Overexpression of an alfalfa GDP-mannose 3, 5epimerase gene enhances acid, drought and salt tolerance in transgenic Arabidopsis by increasing ascorbate accumulation. Biotechnol Lett. 2014; 36(11):2331-41.

26. Fotopoulos V, Sanmartin M, Kanellis AK. Effect of ascorbate oxidase overexpression on ascorbate recycling gene expression in response to agents imposing oxidative stress. J Exp Bot. 2006;57(14):3933-43.

27. Rituraj B, Kapil S, Sumita K, Ananda M. Transcript profiling reveals the presence of abiotic stress and developmental stage specific Ascorbate oxidase genes in plants. Front Plant Sci. 2017;8:198.

28. Anderson JV, Davis DG. Abiotic stress alters transcript profiles and activity of glutathione S-transferase, glutathione peroxidase, and glutathione reductase in Euphorbia esula. Physiol Plant. 2004;120(3):421-33.

29. Alscher RG. Biosynthesis and antioxidant function of glutathione in plants. Physiol Plant. 1989;77(3):457-64.

30. Landi S, Nurcato R, De Lillo A, Lentini M, Grillo S, Esposito S. Glucose-6phosphate dehydrogenase plays a central role in the response of tomato (Solanum lycopersicum) plants to short and long-term drought. Plant Physiol Biochem. 2016;105:79-89.

31. Liu J, Wang $X, \mathrm{Hu}$ Y, Hu W, Bi Y. Glucose-6-phosphate dehydrogenase plays a pivotal role in tolerance to drought stress in soybean roots. Plant Cell Rep. 2013:32(3):415-29.

32. Wagner U, Edwards R, Dixon DP, Mauch F. Probing the diversity of the Arabidopsis glutathione S-Transferase gene family. Plant Mol Biol. 2002;49: 515-32.

33. Xu J, Xiao-Juan X, Yong-Sheng T, Ri-He P, Yong X, Wei Z, Quan-Hong Y, Hong Z. Transgenic Arabidopsis plants expressing tomato glutathione STransferase showed enhanced resistance to salt and drought stress. PLoS One. 2015;10(9):e0136960.

34. Nijveldt RJ, van Nood E, van Hoorn DE, Boelens PG, van Norren $K$, van Leeuwen PA. Flavonoids: a review of probable mechanisms of action and potential applications. Am J Clin Nutr. 2001;74(4):418-25.

35. Wang $P$, Zhang $L$, Jiang $X$, Dai $X, X i a ~ T$. Evolutionary and functional characterization of leucoanthocyanidin reductases from Camellia sinensis. Planta. 2017:247(1):139-54

36. Chen A-H, Chai Y-R, Li J-N, Chen L. Molecular cloning of two genes encoding Cinnamate 4-hydroxylase $(\mathrm{C} 4 \mathrm{H})$ from oilseed rape (Brassica napus). J Biochem Mol Biol. 2007:40(2):247-60. 
37. Rani A, Singh K, Ahuja PS, Kumar S. Molecular regulation of catechins biosynthesis in tea [Camellia sinensis (L.) O. Kuntze]. Gene. 2012;495(2):205-10.

38. Bellés JM, López-Gresa MP, Fayos J, Pallás V, Rodrigo I, Conejero V. Induction of cinnamate 4-hydroxylase and phenylpropanoids in virus-infected cucumber and melon plants. Plant Sci (Oxford). 2008;174(5):524-33.

39. Wang F, Ren G, Li F, Qi S, Xu Y, Wang B, Yang Y, Ye Y, Zhou Q, Chen X. A chalcone synthase gene AeCHS from Abelmoschus esculentus regulates flavonoid accumulation and abiotic stress tolerance in transgenic Arabidopsis. Acta Physiol Plant. 2018;40(5):97.

40. Iracema B, Maria AA, Pedro R, Victor dF, Pedro M-F, Nuno M, Vítor C. Quercetin increases oxidative stress resistance and longevity in Saccharomyces cerevisiae. J Agric Food Chem. 2007;55(6):2446-51.

41. Yamasaki H, SakihamaY IN. Flavonoid-peroxidase reaction as a detoxification mechanism of plant cells against $\mathrm{H}_{2} \mathrm{O}_{2}$. Plant Physiol. 1997;115(4):1405-12.

42. Tattini M, Guidi L, Morassi-Bonzi L, Pinelli P, Remorini D, Degl'Innocenti E, Giordano C, Massai R, Agati G. On the role of flavonoids in the integrated mechanisms of response of Ligustrum vulgare and Phillyrea latifolia to high solar radiation. New Phytol. 2005;167(2):457-70.

43. Lee B-R, Kim K-Y, Jung W-J, Avice J-C, Ourry A, Kim T-H. Peroxidases and lignification in relation to the intensity of water-deficit stress in white clover (Trifolium repens L.). J Exp Bot. 2007;58(6):1271-9.

44. Kang W, Liyuan W, Chengcai Z, Liyun W, Hailin L, Fen Z, Hao C, Kumar MT. Transcriptome analysis reveals key flavonoid 3'-hydroxylase and flavonoid 3,'5'hydroxylase genes in affecting the ratio of Dihydroxylated to Trihydroxylated Catechins in Camellia sinensis. PLoS One. 2015;10(9):e0137925.

45. Jeong ST, Goto-Yamamoto N, Hashizume K, Esaka M. Expression of the flavonoid 3'-hydroxylase and flavonoid 3',5'-hydroxylase genes and flavonoid composition in grape (Vitis Vinifera). Plant Sci. 2006;170(1):61-9.

46. Zhang L-Q, Wei K, Cheng H, Wang L-Y, Zhang C-C. Accumulation of catechins and expression of catechin synthetic genes in Camellia sinensis at different developmental stages. Bot Stud. 2016;57:31.

47. Bogs J, Downey MO, Harvey JS, Ashton AR, Tanner GJ, Robinson SP. Proanthocyanidin synthesis and expression of genes encoding leucoanthocyanidin reductase and anthocyanidin reductase in developing grape berries and grapevine leaves. Plant Physiol. 2005;139(2):652-63.

48. Peng-fei W, Xing-yan N, Yan-fu X, Mei-ying G, Tie-quan N. Spatial and temporal accumulation of Flavanols, activity and tissue localization of Leucoanthocyanidin Reductase induced by soil drought in developing grape berries. Sci Agric Sin. 2013;46(14):2979-89.

49. Song X, Diao J, Ji J, Wang G, Guan C, Jin C, Wang Y. Molecular cloning and identification of a flavanone 3-hydroxylase gene from Lycium chinense, and its overexpression enhances drought stress in tobacco. Plant Physiol Biochem. 2016;98:89-100.

50. Gai Z, Wang Y, Jiang J, Xie H, Ding Z, Ding S, Wang H. The quality evaluation of tea (Camellia sinensis) varieties based on the metabolomics. HortScience. 2019;54(3):409-15.

51. Guo C, Zhang M. Physiological response and molecular basis of tea plant (Camellia sinensis) exposed to water stress. Fujian: Fujian Agriculture And Forestry University; 2008.

52. Su L, Dai Z, Li S, Xin H. A novel system for evaluating drought-cold tolerance of grapevines using chlorophyll fluorescence. BMC Plant Biol. 2015;15(1):82.

53. Xie X, Kang H, Liu W, Wang G-L. Comprehensive profiling of the Rice Ubiquitome reveals the significance of lysine Ubiquitination in young leaves. J Proteome Res. 2015;14(5):2017-25.

54. Di T, Zhao L, Chen H, Qian W, Wang P, Zhang X, Xia T. Transcriptomic and metabolic insights into the distinctive effects of exogenous melatonin and gibberellin on Terpenoid synthesis and plant hormone signal transduction pathway in Camellia sinensis. J Agric Food Chem. 2019;67:4689-99.

55. Yuan H, Zeng X, Shi J, Xu Q, Wang Y, Jabu D, Sang Z, Nyima T. Time-course comparative metabolite profiling under osmotic stress in tolerant and sensitive Tibetian hulless barley. Biomed Res Int. 2018;9415409:1-12.

56. Wang W, Zhou Y, Wu Y, Dai X, Liu Y, Qian Y, Li M, Jiang X, Wang Y, Gao L. An insight into Catechins metabolic pathways of Camellia sinensis based on genome and Transcriptome analysis. J Agric Food Chem. 2018;66(16):4281-93.

57. Pfaff MW. A new mathematical model for relative quantification in real-time RT-PCR. Nucleic Acids Res. 2001;29(9):e45.

\section{Publisher's Note}

Springer Nature remains neutral with regard to jurisdictional claims in published maps and institutional affiliations.

\section{Ready to submit your research? Choose BMC and benefit from}

- fast, convenient online submission

- thorough peer review by experienced researchers in your field

- rapid publication on acceptance

- support for research data, including large and complex data types

- gold Open Access which fosters wider collaboration and increased citations

- maximum visibility for your research: over $100 \mathrm{M}$ website views per year

At BMC, research is always in progress.

Learn more biomedcentral.com/submissions 